\title{
Treatment outcome and associated factors for severely malnourished children (1-5 years) admitted to Lacor Hospital and Gulu Regional Referral Hospital in Uganda
}

\author{
E. Muwanguzi ${ }^{1}$ * (1), J. Eros Oboi ${ }^{2}$, A. Nabbamba ${ }^{2}$ and R. Wanyama ${ }^{3}$ \\ ${ }^{1}$ The Infectious Disease Institute, Kampala, Uganda \\ ${ }^{2}$ Department of Medicine, Gulu University, P.O. Box 166, Gulu, Uganda \\ ${ }^{3}$ Department of Biochemistry, Gulu University, P.O. Box 166, Gulu, Uganda
}

(Received 31 January 2021 - Accepted 12 February 2021)

Journal of Nutritional Science (2021), vol. 10, e33, page 1 of 7

doi:10.1017/jns.2021.11

Abstract

Globally, severe acute malnutrition (SAM) accounts for $>1 / 3-0 \cdot 5$ of deaths in children $<5$ years, and approximately $54 \%$ deaths in developing countries. The minimum international standard set for the management of SAM is a cure rate of at least $75 \%$ and death rate $<10 \%$. The present study was conducted to determine treatment outcome and associated factors among children 1-5 years hospitalised with SAM in Lacor and Gulu Regional Referral Hospital (GRRH) in 2017. A retrospective observational method supplemented with a qualitative inquiry was done. A total of 317 patients' records were reviewed in either hospital; checklist data were analysed using SPSS version 16 with $P$-values $<0.05$ considered for statistical significance. The case fatality rate (CFR) was $12.6 \%(\mathrm{GRRH})$ and $9.5 \%$ (Lacor). The average length of stay (LOS) was 14.69 d (GRRH) and 14.10 d (Lacor). There was statistical significance between Human Immunodeficiency Virus (HIV) status, blood transfusion, type of SAM, treatment provision at admission, antibiotics, mid-upper arm circumference (MUAC), hospital category and treatment outcome. In total, ten key informants were interviewed and they reported the presence of co-infections and severity of SAM complications as having an important bearing on treatment outcome. A significant proportion of patients were discharged not cured $19.9 \%$ (Lacor) $v .16 .4 \%$ (GRRH). The CFR in GRRH was higher than the WHO recommendation. The LOS in both hospitals was within recommended. These results provide a generalisable problem in most African hospitals and could explain the persistently high rates of SAM in Africa.

Key words: Dead: Defaulted: Discharged cured: Not cured: Severe acute malnutrition: Treatment outcome

\section{Introduction}

Globally, severe acute malnutrition (SAM) is associated with poor outcomes. Malnutrition is known to cause growth retardation, impair psychosocial and cognitive development and increase the risk of disease and death. Some studies have shown prolonged hospitalisation ${ }^{(1)}$.

In 2011, out of the thirty-three countries that accounted for $90 \%$ of the global burden of malnutrition, twenty two were from Africa ${ }^{(2)}$. In Uganda, $14 \%$ of children are severely stunted, $2 \%$ are severely wasted and $3 \%$ are severely underweight ${ }^{(3)}$. In Gulu, there is an SAM prevalence of $0.7 \%(0.3-1 \cdot 1 \%)^{(4)}$. Globally, SAM accounts for $>1 / 3-0 \cdot 5$ of all deaths in children under 5 years and approximately $54 \%$ deaths in developing countries ${ }^{(5)}$. The minimum international standard set for the management of SAM is a case fatality rate (CFR) less than $10 \%{ }^{(6)}$.

Abbreviations: CFR: case fatality rate; CI: confidence interval; FANTA-2: Food and Nutrition Technical Assistance 11; GRRH: Gulu Regional Referral Hospital; HIV: Human Immunodeficiency Virus; LOS: length of stay; MUAC: mid-upper arm circumference; PNFP: private not-for-profit; SAM: severe acute malnutrition; sD: standard deviation; TFC: Therapeutic Feeding Center; UDHS: Uganda Demographic and Health Survey; UNICEF: United Nations Children's Fund; WHO: World Health Organisation.

*Corresponding author: E. Muwanguzi, email muwanguzieria@gmail.com

(C) The Author(s), 2021. Published by Cambridge University Press on behalf of The Nutrition Society. This is an Open Access article, distributed under the terms of the Creative Commons Attribution-NonCommercial-ShareAlike licence (http://creativecommons.org/licenses/by-nc-sa/4.0/), which permits non-commercial re-use, distribution, and reproduction in any medium, provided the same Creative Commons licence is included and the original work is properly cited. The written permission of Cambridge University Press must be obtained for commercial re-use. 
However, the case fatality rates in hospitals treating SAM in developing countries have remained high at $20-30 \%{ }^{(7)}$ inspite of existing clinical management protocols capable of reducing these rates ${ }^{(7,8)}$. A retrospective study to determine the treatment outcome of severely malnourished children in St. Mary's Hospital Lacor, Northern Uganda, showed a CFR of $11.9 \%{ }^{(7)}$.

Limited research data exist on treatment outcomes for SAM children admitted to hospitals in northern Uganda. This research aimed to provide data on Gulu Regional Referral Hospital (GRRH) and Lacor Hospital and advice on the minimum average length of stay (LOS) in hospital and Case Fatality Rates and guide hospital management and inpatient care policies so as to address factors that contribute to poor treatment outcome of these children.

\section{Methods}

\section{Study design}

This will be a hospital record-based retrospective observational study supplemented with a qualitative inquiry

\section{Ethical standards disclosure}

Since it was a retrospective study that did not involve human research participants, ethical approval to access the data was provided by the Gulu University Research and Ethics Committee and approvals obtained from the administration of Gulu Regional Referral and Lacor Hospitals. No written or verbal consent was involved.

\section{Study variables}

Dependent variable. Length of hospitalisation, discharge status.

Independent variables. Type of SAM, Human Immunodeficiency Virus (HIV) status, complications of SAM, time of commencement and nature of treatment etc.

\section{Sample size}

The present study was carried out from GRRH and Lacor Hospital. Lacor Hospital is a private not-for-profit (PNFP) entity located in Gulu district $6 \mathrm{~km}$ west of Gulu town along the Highway to Sudan, about $6 \mathrm{~km}$ from GRRH. According to the hospital's manual for 2008/2009, Lacor Hospital saw an influx of 225616 clients treated as inpatients with children below five making up $44.5 \%$ of the total attendance and a CFR of $4.8 \%$. Lacor Hospital has a well-facilitated SAM unit on its paediatric ward where the present study was carried out. GRRH is located in Gulu town and is one of the 13 public regional referral hospitals in Uganda. The hospital serves the Acholi sub-region comprising the districts of Gulu, Amuru, Kitgmu, Lamwo and Pader. The hospital manual for 2008/2009 puts the average bed occupancy rate at 220 and an annual inpatient admission of 15 896. The study was carried out on the SAM unit of the paediatrics ward. Professional cadres providing care include nursing officers, medical doctor interns and medical officers.

The records of severely malnourished children aged 1-5 years previously admitted to hospital from 1 January 2017 to 31 December 2017 were selected from the Therapeutic Feeding Center (TFC) register book and patients' cards obtained. Children who had weight-for-height less than -3 Z-score, nutritional oedema and mid-upper arm circumference (MUAC) $<11.5 \mathrm{~cm}$ were considered Severely Malnourished. For children who defaulted on treatment and those with incomplete hospital records of anthropometric measurements (weight, height, MUAC), demographics were excluded from the study. Children with documented secondary undernutrition, except HIV, e.g. cerebral palsy and those with oedema unrelated to malnutrition were excluded. Patient cards were assigned random numbers with the sample size arrived at by selecting the numbers randomly without replacement. The sample size was estimated using the Keish Leslie formula (1965)

$$
\frac{N=\left(Z^{2} P Q\right)}{D^{2}}
$$

where $N$ is the minimum sample size; $Z=1.96$ (confidence interval of $95 \%) ; P$ is an average cure rate in admitted under-five children with SAM; and it is $75 \%{ }^{(9)} ; Q=1-P$; $D$ is the maximum error (0.05); so, $N=288$, assuming $10 \%$ documents could not be found, sample size will be $288+$ $29=317$.

N.B: 317 was the sample size in each of the two hospitals. Of all the children admitted in 2017, twenty five children in Lacor and fifty eight in GRRH met the exclusion criteria. This number was mostly contributed by incompletely filled records.

For qualitative data, the sample size for the study participants recruited for Key Informant Interview was estimated through the selection of participants until a saturation point was reached. First, a list of staff with their duration of service with SAM was obtained. Only medical personnel with greater than 3 months of service with SAM were selected for the interview. These were interviewed by a team of three medical personnel and a nutritionist until the responses were constant. In total, five key informants were interviewed at each hospital. To ensure consistency in assessment, all members were present during interviews at the two hospitals, with the lead interviewer remaining the same. The checklist for empirical data was adopted from a study on $\mathrm{SAM}^{(7)}$ after review by a nutritionist. The questionnaire for qualitative data was designed by a nutritionist so as to ensure the quality and reliability of information collected.

\section{Statistical analysis}

Information was recorded on a checklist format and data analysed using Statistical Package for Social Scientists (SPSS) version 16. Categorical variables were summarised as frequencies 
and proportions, while continuous variables as means and standard deviations (SD). $\chi^{2}$ test and $t$-test were used to measure the strength of association between the factors considered and the dependent variables. A $P$-value less than 0.05 was considered for statistical significance.

Results

\section{Empirical data}

In Lacor Hospital, 415 children aged 1-5 years were hospitalised in 2017 ( $\nu .378$ at GRRH), and 390 met the selection criteria ( $v .320$ in GRRH) from which a sample size of 317 was raised in either hospital. The percentage of males admitted at either hospital was $59 \%$. Only $18.3 \%(8.8 \%$ in GRRH) were HIV positive and a greater proportion of children had infection at admission 56.5\% (80.8\% at GRRH) (Table 1). Of the 317 SAM children hospitalised, almost two-thirds $70 \cdot 6$ $\%(71 \%$ in GRRH) were successfully discharged as cured $\left(P=0.96, \quad \chi^{2} 0.002\right), \quad 9.5 \%(12.6 \%$ in $\mathrm{GRRH})$ died $\left(P=0.23, \chi^{2} 1.429\right)$ and $19.9 \%$ were not cured $(16.4 \%$ in GRRH) (Table 2). The average LOS (days) was slightly higher in GRRH $14.69 \pm 7.42$ ( $v .14 .1 \pm 7.33$ in Lacor), with patients staying more in the stabilisation phase than in the rehabilitation phase (Table 3). The mean age for the children in GRRH was 24 months (25 in Lacor), while the mean height of children was 80 (77 in Lacor). There was no difference in the mean weight and MUAC between hospitals (Table 3). Overall, the average ages for SAM patients who died, cured and those not cured were 21.7, 24.7 and 26.1 months, respectively (Fig. 1).

Table 4 shows the significant differences in factors compared between GRRH and Lacor Hospital. At multivariate analysis, significant factors associated with treatment outcome (CFR \& LOS) are shown in Table 5.

HIV negative children were more likely to be cured compared with their HIV positive counterparts in both hospitals, GRRH (76.5 v. 14.3\%) and Lacor (74.5 v. 53.4\%); $(P<0.001 ; 95 \%$ CI $0.44-0 \cdot 70)$. HIV positive children were more likely to die compared with their HIV negative counterparts in both hospitals, GRRH (71.4 v. 26.9\%) and Lacor $(44.8 v .1 .5 \%)$ (Table 6).

\section{Qualitative data}

\section{Key informant interviews}

Gulu Regional Referral Hospital. We interviewed staff on the malnutrition unit of GRRH; a total of 5 key informants were interviewed at which the responses to the given questions were no longer differing. Only $20 \%(1 / 5)$ of the respondents were aged between 21 and 30 years, $20 \%(1 / 5)$ aged 31-40 and 60 $\%(3 / 5)$ aged between 41 and 50 years. A total of $60 \%(3 / 5)$ of respondents were male. Professional cadres included in the present study involved two nursing officers, two medical interns and one medical officer. A total of $80 \%$ (4/5) of the respondents had worked for 2-3 months on the paediatric ward, while one nurse had worked for over 10 years.

All health workers reported that the children admitted at the malnutrition unit fulfilled the World Health Organisation
Table 1. Socio-demographic characteristics of participants

\begin{tabular}{|c|c|c|}
\hline \multirow[b]{2}{*}{ Variable } & \multicolumn{2}{|c|}{ Frequency (\%) } \\
\hline & Gulu & Lacor \\
\hline \multicolumn{3}{|c|}{ Discharge status } \\
\hline Cured & $225(71)$ & $224(70.7)$ \\
\hline Died & $40(12.6)$ & $30(9.5)$ \\
\hline Not cured & $52(16.4)$ & $63(19.9)$ \\
\hline \multicolumn{3}{|l|}{ Sex } \\
\hline Female & $135(42 \cdot 6)$ & $130(41.0)$ \\
\hline Male & $182(57.4)$ & $187(59.0)$ \\
\hline \multicolumn{3}{|l|}{ HIV status } \\
\hline Negative & $289(91 \cdot 2)$ & $259(81 \cdot 7)$ \\
\hline Positive & $28(8.8)$ & $58(18.3)$ \\
\hline \multicolumn{3}{|c|}{ Infection at admission } \\
\hline No & $61(19 \cdot 2)$ & $138(43.5)$ \\
\hline Yes & $256(80.8)$ & $179(56.5)$ \\
\hline \multicolumn{3}{|c|}{ Vitamin A given } \\
\hline No & $17(5.4)$ & $41(12.9)$ \\
\hline Yes & $300(94.6)$ & $276(87 \cdot 1)$ \\
\hline \multicolumn{3}{|l|}{ Zinc given } \\
\hline No & $99(31.2)$ & $105(33 \cdot 1)$ \\
\hline Yes & $218(68.8)$ & $212(66.9)$ \\
\hline \multicolumn{3}{|c|}{ Antibiotic given } \\
\hline No & $26(8 \cdot 2)$ & $43(13.6)$ \\
\hline Yes & $291(91 \cdot 8)$ & $274(86.4)$ \\
\hline \multicolumn{3}{|c|}{ Iron given at admission } \\
\hline No & $317(100)$ & $283(89.3)$ \\
\hline Yes & $0(0)$ & $34(10.7)$ \\
\hline \multicolumn{3}{|c|}{ Dehydration at admission } \\
\hline No & $55(17.4)$ & $89(28.1)$ \\
\hline Yes & $262(82.6)$ & $228(71.9)$ \\
\hline \multicolumn{3}{|c|}{ Hypoglycaemia at admission } \\
\hline No & $231(72.9)$ & $190(59.9)$ \\
\hline Yes & $86(27 \cdot 1)$ & $127(40.1)$ \\
\hline \multicolumn{3}{|c|}{ Severe anaemia at admission } \\
\hline No & $198(62 \cdot 5)$ & $229(72 \cdot 2)$ \\
\hline Yes & $119(37.5)$ & $88(27.8)$ \\
\hline \multicolumn{3}{|c|}{ Treatment given at admission } \\
\hline No & $159(50 \cdot 1)$ & $258(81.4)$ \\
\hline Yes & $158(49.9)$ & $59(19.6)$ \\
\hline \multicolumn{3}{|c|}{ Hypoglycaemia treated at admission } \\
\hline No & $140(44 \cdot 2)$ & $185(58.4)$ \\
\hline Yes & $177(55.8)$ & $132(41.6)$ \\
\hline \multicolumn{3}{|c|}{ Dehydration treated at admission } \\
\hline No & $35(11.0)$ & $77(24.3)$ \\
\hline Yes & $282(89.0)$ & $240(75.7)$ \\
\hline \multicolumn{3}{|c|}{ Hypothermia treated at admission } \\
\hline No & $165(52 \cdot 1)$ & $170(53.8)$ \\
\hline Yes & $152(47.9)$ & $147(46 \cdot 2)$ \\
\hline
\end{tabular}

(WHO) definition of SAM (having either an MUAC $<11.5$ cm or nutritional oedema $\left.{ }^{(10)}\right)$.

All respondents, $100 \%$ (5/5), reported that daily admissions ranged from 1 to 4 , with one respondent reporting that this depended on the season of the year: 'The daily admission

Table 2. Comparison of the status at discharge in GRRH and Lacor Hospital

\begin{tabular}{lcrl}
\hline & \multicolumn{2}{c}{ Frequency $(\%)$} \\
\cline { 2 - 3 } Status at discharge & Lacor Hospital & GRRH & \\
\hline Cured & $224(70.6)$ & $225(71.0)$ & $0.002,0.96$ \\
Died & $30(9.5)$ & $40(12.6)$ & $1.429,0.23$ \\
Not cured & $63(19.9)$ & $52(16.4)$ & $1.052,0.31$ \\
\hline
\end{tabular}


Table 3. Comparison of mean \pm standard deviation of selected continuous variables between Lacor and Gulu Regional Referral Hospitals

\begin{tabular}{|c|c|c|c|}
\hline Variable & Hospital & Mean \pm SD & Min-max \\
\hline \multirow[t]{2}{*}{$\operatorname{LOS}(d)$} & Gulu & $14.69 \pm 7.42$ & $1-45$ \\
\hline & Lacor & $14 \cdot 10 \pm 7 \cdot 33$ & $2-57$ \\
\hline \multirow[t]{2}{*}{ Duration in rehabilitation phase $(\mathrm{d})$} & Gulu & $4.76 \pm 3.36$ & $0-18$ \\
\hline & Lacor & $5.96 \pm 4.64$ & $0-32$ \\
\hline \multirow{2}{*}{ Duration in stabilisation phase $(\mathrm{d})$} & Gulu & $9.81 \pm 5.01$ & $1-31$ \\
\hline & Lacor & $8.04 \pm 4.14$ & $1-35$ \\
\hline \multirow[t]{2}{*}{ Age (years) } & Gulu & $24.26 \pm 13.01$ & $12-60$ \\
\hline & Lacor & $25.03 \pm 12.97$ & $12-60$ \\
\hline \multirow[t]{2}{*}{ Height $(\mathrm{cm})$} & Gulu & $80 \cdot 28 \pm 12 \cdot 42$ & $59-115$ \\
\hline & Lacor & $77.73 \pm 11.89$ & $53-121$ \\
\hline \multirow[t]{2}{*}{ Weight (kg) } & Gulu & $8.47 \pm 1.87$ & $4-16$ \\
\hline & Lacor & $8.47 \pm 2.37$ & $4-20$ \\
\hline \multirow[t]{2}{*}{ Muac (cm) } & Gulu & $11.09 \pm 1.04$ & $9 \cdot 0-14 \cdot 6$ \\
\hline & Lacor & $11.07 \pm 1.15$ & $6 \cdot 5-16 \cdot 5$ \\
\hline
\end{tabular}

depends on the season, e.g. every year, March through July, admissions are higher.'

The respondents reported a variable time of initiation of treatment of SAM children from admission, with $40 \%(2 / 5)$ reporting that treatment was initiated immediately on admission and $40 \%(2 / 5)$ reporting that it was initiated after $1 \mathrm{~h}$ from admission, with only $20 \%(1 / 5)$ reporting that the time depended on the workload and the health workers present to give treatment at a particular time.

All respondents unanimously stated that feeding formulas and medication were available. All of them, however, reported that the availability of medication varied throughout the month, with one key informant reporting that, 'Drugs are present at the start of the month but decrease towards the end.'

All of the respondents, $100 \%(5 / 5)$, reported about the inadequacy of investigative and diagnostic capacity in the hospital, with two of them saying that, 'Most times machines in the lab are down,' and another emphasising on the inadequacy of facilities and the challenges being faced by the lab in managing SAM. Yet another reported that, 'Not all of the diagnostic equipment are available.'

All respondents reported that, while on ward, all SAM patients are regularly monitored and followed up and that
Table 4. Comparison of the selected continuous variables between the two hospitals

\begin{tabular}{lrrrr}
\hline & \multirow{2}{c}{$\begin{array}{c}\text { Mean } \\
\text { difference }\end{array}$} & $P$-value & Upper & Lower \\
\cline { 4 - 6 } Variable & 1.77 & 0.00 & 1.06 & 2.49 \\
\hline $\begin{array}{l}\text { Duration in stabilisation } \\
\text { phase }\end{array}$ & & & & \\
$\begin{array}{l}\text { Duration in rehabilitation } \\
\text { phase }\end{array}$ & -1.20 & 0.00 & -1.83 & -0.57 \\
$\begin{array}{l}\text { Age } \\
\text { Height }\end{array}$ & -0.78 & 0.46 & -2.80 & 1.26 \\
Weight & 2.55 & 0.01 & 0.65 & 0.45 \\
Muac & 0.00 & 0.99 & -0.33 & 0.34 \\
\hline
\end{tabular}

patients are discharged only after appropriate weight gain and the attainment of a good appetite.

Five out of five $(100 \%)$ of the respondents reported a longer period of stay for SAM patients compared with their counterparts hospitalised for other conditions without SAM, with one reporting that, 'SAM patients spend 21 days, other conditions about five days.' Another respondent reported a range of $2-4$ weeks for SAM patients compared with their counterparts who spend about $2-5 \mathrm{~d}$ in hospital.

There was a unanimous response to the availability of training sessions for health workers, with all of the respondents stating that they are available and are regularly done.

All respondents commented on a prolonged LOS and poor discharge status for SAM children admitted with co-infections. They also agreed that this can be improved by aggressive treatment of these co-infections.

During our interview, responses on how to improve the outcome of SAM children that were suggested by key informants included: early initiation of treatment stated in only $12.5 \%(1 / 8)$ responses, $50 \%(4 / 8)$ responses advised on adequate supply of medications and availability of diagnostics, 2/8 (25\%) advocated on community sensitisation on nutrition health education and counselling with increased workforce, while one respondent emphasised on the treatment of co-infections.

Lacor. A total of 5 key informants were interviewed, with $60 \%$ (3/5) of the respondents aged between 21 and 30 years, $20 \%$

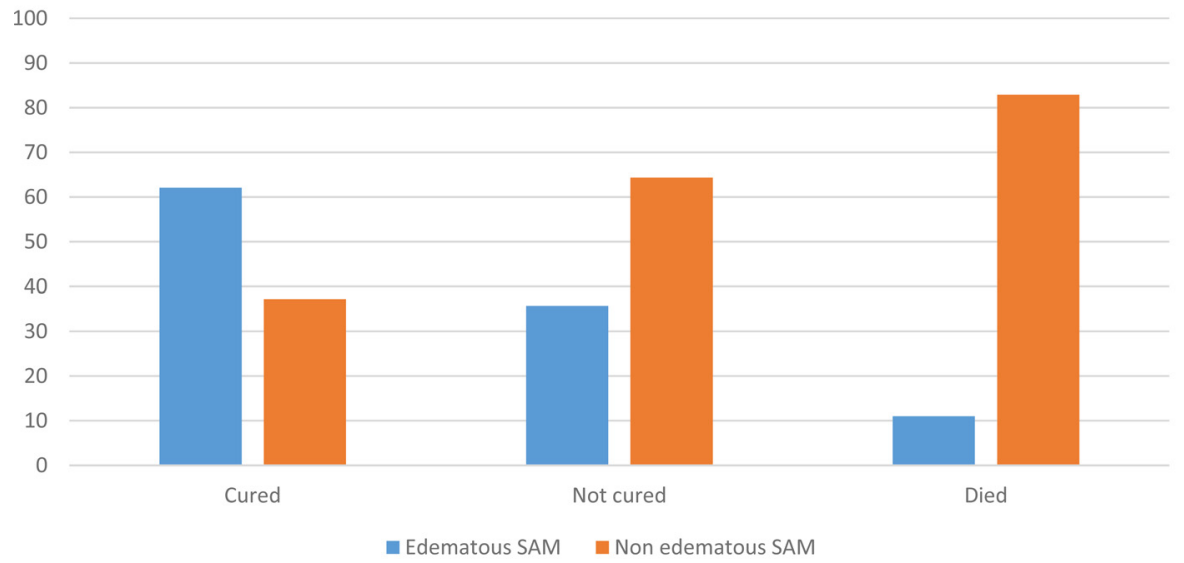

Fig. 1. Treatment outcome per SAM type. 
Table 5. Factors associated with treatment outcome

\begin{tabular}{lrrr}
\hline & & \multicolumn{2}{c}{$95 \% \mathrm{Cl}$} \\
\cline { 3 - 4 } Variable & & Lower & Upper \\
\hline CFR & & & \\
Vitamin A & 0.67 & -0.12 & 0.19 \\
Antibiotic given & 0.00 & 0.27 & 0.55 \\
Muac & 0.01 & -0.09 & -0.01 \\
Sex & 0.00 & 0.07 & 0.24 \\
HIV status & 0.00 & 0.44 & 0.70 \\
Hospital category & 0.03 & -0.20 & -0.10 \\
Blood transfusion & 0.00 & -0.52 & -0.31 \\
SAM type & 0.00 & 0.22 & 0.40 \\
Treatment at admission & 0.00 & 0.07 & 0.26 \\
Infection at admission & 0.00 & -0.28 & -0.08 \\
LOS & & & \\
MUAC & 0.17 & -0.92 & 0.16 \\
HIV status & 0.10 & -3.16 & 0.27 \\
Vitamin A supplementation & 0.03 & -4.24 & -0.22 \\
Antibiotic given & 0.02 & -4.07 & -0.35 \\
Iron supplementation & 0.03 & -5.43 & -0.31 \\
SAM type & 0.02 & -2.59 & -0.21 \\
\hline
\end{tabular}

(1/5) aged 31-40 and 20\% (1/5) aged between 41 and 50 years. A total of $80 \%(4 / 5)$ were females. A total of $60 \%$ $(3 / 5)$ were intern doctors, and only $20 \%(1 / 5)$ were nurses and one medical officer. A total of $80 \%$ (4/5) of the respondents had worked in a paediatric ward for a duration of 1-6 months, while one had a duration of 1 year.

Two-fifths $(40 \%)$ of the responses included the presence of nutritional oedema in children to be managed as cases of SAM, $40 \%(4 / 10)$ stated a Muac of $<11.5 \mathrm{~cm}$, and one respondent included the presence of complications associated with SAM (such as hypoglycaemia) for children to be admitted.

A majority of respondents (3/5) reported daily admissions ranging between 6 and 10, with $40 \%$ reporting about $1-5$ daily admissions.

There were differences in the time of initiation of treatment on admission, with three respondents stating $1 \mathrm{~h}$, while the other two stating $30 \mathrm{~min}$.

All respondents reported about the availability of feeding formulas, medications, and good diagnostic facilities and about the fact that patients are monitored regularly, with one respondent stating that, 'Nurses are ever around during serving and feeding of children.'

The responses on the criteria for discharge of SAM patients were varied and included weight gain as suggested by $40 \%$ $(2 / 5)$ of the respondents, patients who pass the appetite test, $40 \%(2 / 5)$, and those who complete the resolution of nutritional oedema, which was suggested by $20 \%(1 / 5)$ respondents.

Table 6. Relationship between HIV status and discharge status in Lacor and Gulu Regional Referral Hospitals

\begin{tabular}{lllll}
\hline & & \multicolumn{3}{c}{ Discharge status (frequency) } \\
\cline { 3 - 5 } HIV status & Hospital & Cured & Died & Not cured \\
\hline Positive & Lacor & $31(53.4 \%)$ & $26(44.8 \%)$ & $1(1.7 \%)$ \\
& GRRH & $4(14.3 \%)$ & $20(71.4 \%)$ & $4(14.3 \%)$ \\
Negative & Lacor & $193(74.5 \%)$ & $4(1.5 \%)$ & $62(23.9 \%)$ \\
& GRRH & $221(76.5 \%)$ & $20(26.9 \%)$ & $48(16.6 \%)$ \\
\hline
\end{tabular}

There was a unanimous response suggesting an increased LOS in hospital for SAM patients compared with their counterparts of the same age admitted to other wards.

All respondents reported on the availability of training sessions leading to an improvement in the condition of SAM patients, with one reporting that, 'Yes, they are available around 1 every 3 months.'

All respondents reported that co-infections prolong the LOS of SAM children in hospital and result in poor discharge status.

There was a reported link between the LOS and discharge status of SAM children, and an aggressive management of co-infections and SAM, in general, increased the chances of survival, as reported by all of the respondents.

Concerning the improvement of treatment outcome for SAM patients, about $40 \%(2 / 5)$ of the respondents suggested an increase in the frequency of training sessions on SAM management, $33 \%(2 / 5)$ of the respondents recommended immediate initiation of treatment on admission with direct patient assessment at the malnutrition unit, and $20 \%(1 / 5)$ of the respondents suggested that health workers should be motivated through better pay and other kinds of remuneration.

\section{Discussion}

The average LOS in GRRH was $14.69 \mathrm{~d}$ (14.10 in Lacor) $(P=$ $0 \cdot 31$; CI -0.56 to 1.75$)$. This is within the WHO recommendation of less than $30 \mathrm{~d}$ for children hospitalised with SAM. This indifference in LOS between the hospitals could be explained by the similarity in age, sex distribution and anthropometric measurements of the study participants together with the common adoption of the WHO management protocol for SAM children by both hospitals. A study done in Mulago Uganda ${ }^{(1)}$ reported a higher LOS; this could be because the study included patients with other chronic causes of SAM like cerebral palsy. Despite the satisfactory average LOS, the maximum duration of stay in hospital was long; $45 \mathrm{~d}$ in GRRH (57 in Lacor). This is because both sites are referral centres with a heterogeneity of cases.

The CFR was 12.6 and 9.5\% in GRRH and Lacor Hospital, respectively. This is regarded as poor and moderate, respectively ${ }^{(11)}$. However, the present study reported a reduction in the CFR of 2.4 from that depicted by the findings of Nyeko et al. ${ }^{(7)}$. This may be attributed to an increased health workforce and diagnostic capacity at Lacor Hospital over the year. The high CFR in GRRH agrees with the findings of Bartz et al. ${ }^{(1)}$ in Mulago, Uganda. Resource availability may need to be standardised in both hospitals so as to facilitate the best quality of SAM care.

Though the difference in CFR between the hospitals was not statistically significant $\left(P=0.23, X^{2} 1.429\right)$, the higher CFR in GRRH is explained by the inconsistency in the availability of diagnostic modalities for life-threatening comorbidities like severe anaemia, as noted by the responses of the key informants from GRRH, and the higher retention of patients in hospital since services are free of charge. This is in agreement with the results reported by Burns et al. ${ }^{(12)}$ in the USA, depicting a generally increased mortality for patients treated in government hospitals compared with private aided hospitals. 
A high proportion of SAM children were not cured; $16.4 \%$ in GRRH (19.9 \% in Lacor) (Table 2). This could be explained by a higher proportion of HIV-SAM co-infection among children hospitalised causing more clinical complications ${ }^{(7)}$. In addition to the fact that Lacor Hospital is a private not-for-profit institution that imposes medical service charges and hence patients may opt for self-discharge before completing treatment, the hospital also caters to a wider population (including distant referrals from other regions of the country) compared with GRRH, and this could also explain the slightly higher non-cure rate there.

Factors significantly associated with CFR include the following: HIV status, blood transfusion, the type of SAM, treatment provision at admission, sex, antibiotics administration, MUAC patients at admission, the hospital of treatment and the time of treatment initiation from admission. The association of HIV status with SAM treatment outcome is in keeping with the findings of Nyeko et al. ${ }^{(7)}$ at Lacor Hospital, Uganda. A study by Blossner and Onis ${ }^{(13)}$ also showed a significant relationship between the severity of anthropometric deficits and the risk of mortality among SAM patients. The association between CFR and blood transfusion is in agreement with the findings by Gebremichael $e t a l^{(14)}$ that showed increased deaths in children who received blood transfusions. The same study demonstrated the significance of antibiotics on treatment outcome. Vitamin A supplementation was not independently associated with mortality in SAM patients at multivariate analysis. This is in agreement with the findings by Smith et al. ${ }^{(15)}$ in Tanzania $(P=0.54$; CI $0.92-1 \cdot 16)$.

Factors significantly associated with LOS include: SAM type, antibiotic administration, and vitamin $\mathrm{A}$ and iron supplementation during the rehabilitation phase. Children with oedematous SAM usually have poor appetite, reduced food intake and recovery time. This was also in agreement with a study by Agarwal et al. ${ }^{(16)}$ in which the SAM type affected the LOS, the frequency of readmissions and in-hospital CFR. Antibiotic provision at admission reduces infection and LOS; this is in line with the findings of Chisti et al. ${ }^{(17)}$. The study found an association between Vitamin A supplementation and LOS similar to a study by Lannotti et al..$^{(18)}$ that explained the role of vitamin A in re-epithelialization of body surfaces and boosting immunity. LOS was not independently affected by MUAC and HIV status, and this was also in keeping with findings ${ }^{(13)}$.

\section{Conclusion}

The average LOS for SAM children in GRRH and Lacor of 14.69 and $14.10 \mathrm{~d}$, respectively, is satisfactory compared with the WHO recommendation of less than $30 \mathrm{~d}$. The CFR of $12.6 \%$ for children hospitalised in GRRH is still higher than the WHO recommendation of less than $10 \%$. However, the mortality of $9.5 \%$ in Lacor is fair. At both Lacor and GRRH, a significant proportion of patients with SAM are discharged not cured (19.9 v. $16.4 \%)$, a portion of which is contributed by those who opt for discharge before treatment completion, as well as non-response to treatment. The CFR for children hospitalised with SAM in both hospitals is still high, an issue of public health concern.
HIV infection contributed significantly to mortality in children hospitalised with SAM in addition to the type of SAM, iron and vitamin A supplementation, antibiotic administration, MUAC, the presence of co-infection and the time of treatment initiation all at admission, hospital category and blood transfusion.

Facilitation in SAM is key to good treatment outcomes.

\section{Recommendations}

Further research on the effect of Viral Load and CD4 cell count for SAM patients at admission on treatment outcome: With this, we recommend routine CD4 and Viral load for all HIV patients admitted with SAM, a guideline for Sub-Saharan Africa.

Proper home-based care guidelines for children with SAM should be drafted and communities sensitised.

\section{Study limitation}

Recording bias of secondary data by health professionals.

Being a retrospective study, other factors that have an important bearing on treatment outcome, including biochemical laboratory parameters and health system factors (diagnostic capacity, knowledge and skills, referral system and patient monitoring) among others, were analysed only by hearsay (Key Informants) in this study.

\section{Acknowledgements}

This research was possible, thanks to support from Gulu University Faculty of Medicine and the staff at GRRH and the Lacor hospital malnutrition unit during the year 2018.

This research received no specific grant from any funding agency in the public, commercial or not-for-profit sector.

All authors designed the study and coordinated the data management. E. M., E. J. O. and A. N. obtained funding and obtained the data. R. W. prepared the data and did the statistical analyses. All authors interpreted the statistical analyses, wrote the manuscript, critically reviewed the paper and approved the final version.

The authors declare that they have no conflicts of interest.

\section{References}

1. Bartz S, Mody A, Bain J, et al. (2014) Severe acute malnutrition in childhood: hormonal and metabolic status at presentation, response to treatment, and predictors of mortality. J Clin Endocrinol Metab 99, $2128-2137$.

2. de Onis M (2012) The Burden of Malnutrition in Africa. Geneva: WHO.

3. Uganda Bureau of Statistics, ICF International, Inc (2017) Uganda Demographic and Health Survey 2016. Kampala, Uganda; Calverton, MD: Uganda Bureau of Statistics; ICF International, Inc; available at www.health.go.ug/download/file/fid/1325.

4. UNICEF. (HAS NO AUTHOR) (2011). Nutrition Surveillance Acholi Region, Uganda.

5. Bain LE, Awah PK, Geraldine N, et al. (2013). Malnutrition in sub-Saharan Africa: burden, causes and prospects. Pan African Med J 15, 120. 
6. Golden Michael \& Grellety Yvonne (2004) Protocol for the Management of Severe Acute Malnutrition. Ethiopia: Federal Ministry of Health, 2007.

7. Nyeko R, Valeria C, Boniface OS, et al. (2016) Treatment outcome among children under 5 years hospitalized with severe acute malnutrition in Lacor Hospital. Biomed Central Nutr 2, 19.

8. Ahmed T, Ali M \& Ullah MM (1999) Mortality in severely malnourished children with diarrhea and use of standardized management protocol. Lancet 353, 1919-1922.

9. Teferi, E, Meskele L, Sahle S, et al. (2010) Treatment outcome of children with severe acute malnutrition admitted to therapeutic feeding centers in Southern region of Ethiopia. Ethiop J Health Dev 24(3), 234-238.

10. World Health Organization. (HAS NO AUTHOR) (2019).

11. Benyera O \& Hyera FLM (2013) Outcomes in malnourished children at a tertiary hospital in Swaziland after implementation of the World Health Organization treatment guidelines. South Africa J Child Health 7, 135-138.

12. Burns RN, Nichols LO, Graney MJ, et al. (1997) Mortality in a public and a private: the severity of antecedent disorders in Medicare patients. Am J Public Health 83, 969-970.
13. Blossner M \& Onis DM (2003). WHO global database on child growth and malnutrition. Int J Epidemiol 32, 518-526.

14. Gebremichael M, Afework MB \& Mache T (2014) Treatment outcome and associated risk factors of severely malnourished under five children admitted to therapeutic feeding centers of Mekelle city, Northern Ethiopia. OALib J 1.

15. Smith ER, Muhihi A, Mshamu S, et al. (2016) The effect of neonatal vitamin A supplementation on morbidity and mortality at 12 months. Int J Epidemiol 45, 2112-2121.

16. Agarwal E, Ferguson M, Banks M, et al. (2013) Malnutrition and poor food intake are associated with prolonged hospital stay, frequent readmissions and greater in hospital mortality. Clin Nutr 32, 737-745.

17. Chisti MJ, Terbruegge M, Vicente LS, et al. (2009) Pneumonia in severely malnourished children in developing countries - Mortality risk, aetiology and validity of WHO clinical signs: a systematic review. Trop Med Int Health 14(10), 1173-1189. doi:10.1111/j.1365-3156.2009. 02364.x.

18. Lannotti LL, Trehan I \& Manary MJ (2013) Review of the safety and efficacy of vitamin A supplementation in the treatment of children with severe acute malnutrition. Nutr J 2013, 125. 